\title{
Role of Ischemia Modified Albumin in Diagnosis of Pulmonary Embolism
}

\author{
Ashraf Abd El Halim ${ }^{1}$, Adel Attia ${ }^{1 *}$, Saad Samra ${ }^{1}$, Tayseer Zytoun ${ }^{2}$, \\ Hosam Eldeen Salah ${ }^{3}$, Manal Al-Anazi ${ }^{4}$ \\ ${ }^{1}$ Chest Department, Faculty of Medicine, Zagazig University, Zagazig, Egypt \\ ${ }^{2}$ Critical Care Department, Faculty of Medicine, Alexandria University, Alexandria, Egypt \\ ${ }^{3}$ Clinical Pathology Department, Faculty of Medicine, Zagazig University, Zagazig, Egypt \\ ${ }^{4}$ Resident Internal Medicine, King Fahad Specialist Hospital, Dammam, KSA \\ Email: adelattia68@yahoo.com
}

Received January 16, 2014; revised February 7, 2014; accepted February 15, 2014

Copyright (C) 2014 Ashraf Abd El Halim et al. This is an open access article distributed under the Creative Commons Attribution License, which permits unrestricted use, distribution, and reproduction in any medium, provided the original work is properly cited. In accordance of the Creative Commons Attribution License all Copyrights (C) 2014 are reserved for SCIRP and the owner of the intellectual property Ashraf Abd El Halim et al. All Copyright (C) 2014 are guarded by law and by SCIRP as a guardian.

\section{ABSTRACT}

Pulmonary embolism is a common and potentially lethal condition. Clinical signs and symptoms for pulmonary embolism are nonspecific. New and simple tests are therefore needed in order to help in early diagnosis of pulmonary embolism. The aim of this work is to elucidate the role of IMA in the diagnosis of pulmonary embolism. Subjects and Methods: 75 patients with suspected pulmonary embolism and 20 control healthy subjects were included in this study. Measurement of IMA was done in all subjects. Results: The mean values of IMA were statistically significantly higher among the PE patient group (0.43 \pm 0.104 ABSU) in comparison with non PE patient group (0.27 $\pm 0.053 \mathrm{ABSU})$ and healthy control subjects $(0.21 \pm \mathbf{0 . 0 8 0} \mathrm{ABSU})$. At cut-off value of 0.305 ABSU, IMA had $97.5 \%$ sensitivity and $71.42 \%$ specificity. The area under the curve was 0.952 . The positive predictive value of this cut-off value was $79.59 \%$ while the negative predictive value was $96.15 \%$. Conclusions: IMA is a good alternative to D-dimer in the diagnosis and exclusion of PE. Larger studies are needed to augment our results.

\section{KEYWORDS}

Ischemia Modified Albumin; D-Dimer; Pulmonary Embolism

\section{Introduction}

Pulmonary embolism (PE) is a common and potentially lethal condition. Most patients who succumb to pulmonary embolism do so within the first few hours of the event. Acute pulmonary embolism is responsible for 100,000 to 300,000 deaths per year in the United States alone. Despite diagnostic advances, delays in pulmonary embolism diagnosis are common and represent an important issue [1]. Clinical signs and symptoms for pulmonary embolism are nonspecific; therefore, patients suspected of having pulmonary embolism because of unexplained dyspnea, tachypnea, or chest pain or the presence of risk factors for pulmonary embolism must undergo diagnostic tests until the diagnosis is ascertained

${ }^{*}$ Corresponding author. or eliminated or an alternative diagnosis is confirmed. So new and simple tests are therefore needed in order to help in early diagnosis of pulmonary embolism [2].

The N-terminal portion of human serum albumin (HSA) is a binding site for transition metal ions such as cobalt, copper and nickel. During ischemia, several changes occur in the amino-terminus of albumin, which result in a significant change in the ability of albumin to bind transition metals, notably, cobalt. Therefore, an assay measuring ischemia modified albumin (IMA) represents a promising marker for the identification of patients with hypoxemia and ischemia [3].

Many studies found that IMA is a sensitive marker in different ischemic conditions like myocardial ischemia, stroke and mesenteric ischemia [4-9]. 


\section{Aim of the Work}

The aim of this work was to elucidate the role of IMA in the diagnosis of pulmonary embolism.

\section{Subjects and Methods}

This study was conducted at the King Fahad Hospital, Saudi Arabia. The study was approved by Ethics and Research Committee. This study included 75 patients with suspected pulmonary embolism and 20 control healthy subjects. All patients and control subjects gave their written informed consent before participating in the study. Patients were divided into two groups:

Group 1: It included 40 patients with proven pulmonary embolism;

Group 2: It included 35 patients which were negative for pulmonary embolism.

\subsection{Exclusion Criteria}

Exclusion criteria were other acute ischemic diseases such as acute coronary syndrome (ACS), acute ischemic cerebrovascular disease, acute peripheral arterial occlusion, or acute mesenteric ischemia; an abnormal serum albumin level making the determination of IMA levels impossible (normal level 3.5 - $5.5 \mathrm{mg} / \mathrm{dl}$ ); advanced liver, kidney or heart failure; age $<18$ years; allergy to contrast material and refusal to participate in the study. All subjects include in this study were submitted for the followings:

- Full history taking: Thorough medical examination, Wells and Geneva scores were calculated, Plain chest $\mathrm{X}$-ray, Arterial blood gases analysis, ECG, Complete blood count (CBC) and differential cell count, liver function tests and kidney function tests.

- Measurement of D-dimer: The D-dimer test was performed using the immunoturbidimetric assay, STALiatest D-DI (Diagnostica Stago, Paris, France). A cut-off value of $500 \mathrm{ng} / \mathrm{ml}$ was selected as the upper limit to exclude thrombosis. All samples were processed and analyzed within 1 - 2 hours of collection [10].

- Measurement of IMA: Measurement of IMA is done by albumin cobalt binding (ACB) assay (Alere (Inverness Medical), Stockport, UK). The assay measures the cobalt binding capacity of albumin in a sample. A known amount of cobalt is added to a patient serum sample. Dithiothreitol (DTT) is added which binds colorimetric change is measured spectrophotometrically the results were reported as absorbance units (ABSU) [11].

- Spiral CT angiography of the chest was done for patient groups (high D-dimer and high clinical probability patients).
This study used a diagnostic approach consisting of a clinical decision rule, D-dimer testing, and chest CT to evaluate patients with suspected PE [12] (Figure 1).

\subsection{Statistical Analysis}

Statistical analysis was performed with the Statistical Package for the Social Sciences, version 16 for Windows (SPSS Inc., Chicago, IL, USA). The differences between groups were tested by one-way analyses of variance (ANOVA) test. Correlations were investigated by means of the Pearson correlation coefficient. Values of $p<0.05$ were considered statistically significant.

\section{Results}

Seventy five patients with suspected PE were included in this work. Pulmonary embolism was confirmed in 40 patients and was excluded in 35 patients.

Baseline characteristics of the PE group and non PE group and risk factors among both groups were shown in Table 1.

In this study the mean values of D-dimer were statistically significantly higher ( $p$ value $<0.001$ ) among the PE patient group (2652 $\pm 2369.37 \mathrm{ng} / \mathrm{ml})$ in comparison with non PE patient group (674 $\pm 532.72 \mathrm{ng} / \mathrm{ml}$ ) and healthy control subjects $(147 \pm 83 \mathrm{ng} / \mathrm{ml})$ (Table 2 and Figure 2).

D-dimer was high at $78.66 \%$ of the patients and was normal at $21.34 \%$ of the patients. D-dimer testing in this study had $97.50 \%$ sensitivity and $42.85 \%$ specificity. The positive predictive value of D-dimer in this study was $66.10 \%$ while the negative predictive value was $93.75 \%$.

In this study the mean values of IMA were statistically

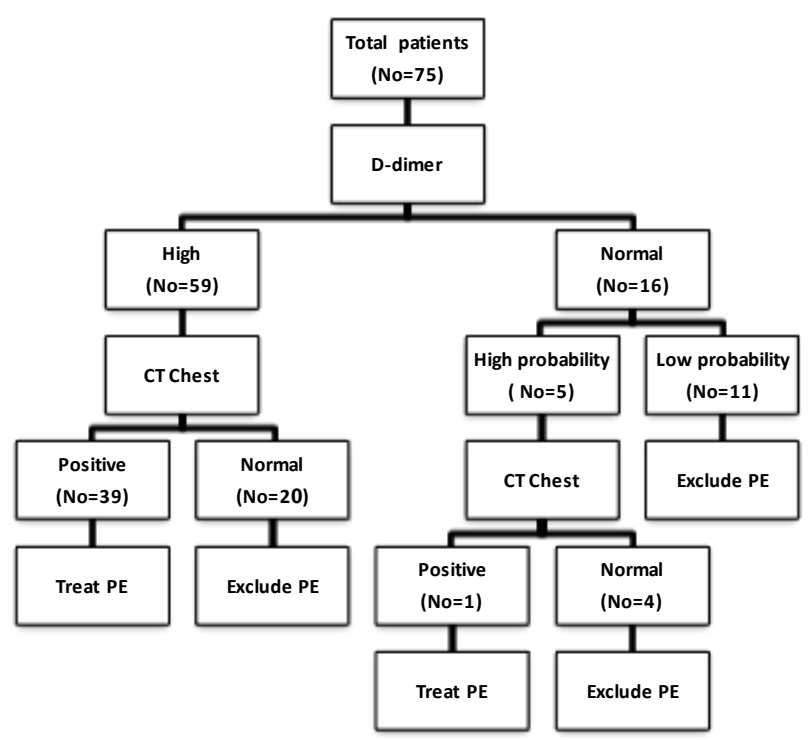

Figure 1. The diagnostic outcome for the subjects included in this study. 
Table 1. Basal characteristic data for the subjects included in this study.

\begin{tabular}{lccc}
\hline & PE group & Non PE group & $\begin{array}{c}\text { Healthy } \\
\text { Subjects }\end{array}$ \\
\hline Sex (male/female) & $23 / 17$ & $19 / 16$ & $12 / 8$ \\
Age (mean \pm SD) & $61.47 \pm 13.78$ & $55.62 \pm 20.03$ & $57.36 \pm 18.65$ \\
Previous PE & 2 & 1 & 0 \\
Previous DVT & 5 & 1 & 0 \\
PaO $_{2}$ mm Hg & $65.30 \pm 5.32$ & $79.51 \pm 6.47$ & $95.50 \pm 3.55$ \\
PaCO $_{2}$ mm Hg & $31.73 \pm 6.20$ & $39.50 \pm 4.81$ & $38.35 \pm 2.75$ \\
$\mathrm{O}_{2}$ saturation\% & $86 \pm 7.5$ & $93 \pm 3.43$ & $97 \pm 2.10$ \\
Associated conditions & & & 0 \\
Cancer & 7 & 6 & 0 \\
COPD & 13 & 11 & 0 \\
Fracture & 2 & 2 & 0 \\
Pregnancy & 1 & 2 & 0 \\
Smoking & 12 & 15 & 0 \\
Surgery & 5 & & 0 \\
Trauma & 5 & & 0 \\
\hline
\end{tabular}

Table 2. IMA and D-dimer mean levels among the subjects included in this study.

\begin{tabular}{ccccc}
\hline Measurements & PE patients & $\begin{array}{c}\text { Non PE } \\
\text { patients }\end{array}$ & $\begin{array}{c}\text { Healthy } \\
\text { Subjects }\end{array}$ & p value \\
\hline IMA (ABSU) & $0.43 \pm 0.104$ & $0.27 \pm 0.053$ & $0.21 \pm 0.080$ & $<0.001$ \\
D-dimer (ng/ml) & $2652 \pm 2369.37$ & $674 \pm 532.72$ & $147 \pm 83$ & $<0.001$ \\
\hline
\end{tabular}

ABSU: absorbance units.

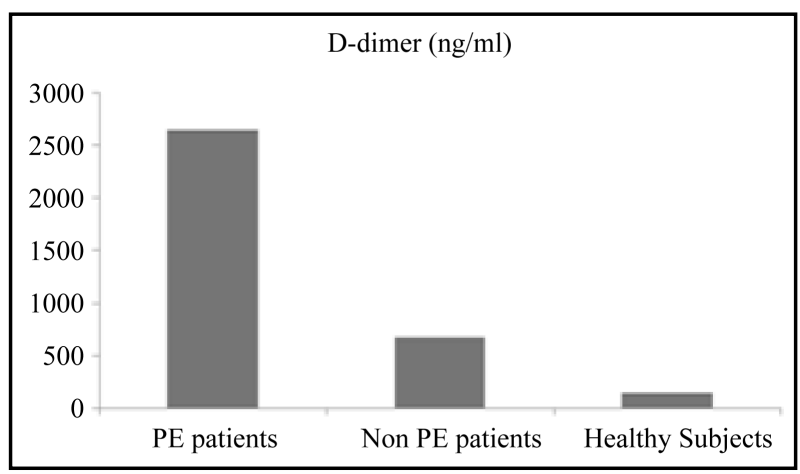

Figure 2. D-dimer mean levels among the subjects included in this study.

significantly higher ( $p$ value $<0.001$ ) among the PE patient group $(0.43 \pm 0.104 \mathrm{ABSU})$ in comparison with non $\mathrm{PE}$ patient group $(0.27 \pm 0.053 \mathrm{ABSU})$ and healthy control subjects $(0.21 \pm 0.080$ ABSU) (Table 2 and Figure 3). At cut-off value of 0.305 ABSU, IMA had $97.5 \%$ sensitivity and $71.42 \%$ specificity. The area under the curve was 0.952 . The positive predictive value of this cut-off value was $79.59 \%$ while the negative predictive value was 96.15\% (Figure 4).
IMA (ABSU)

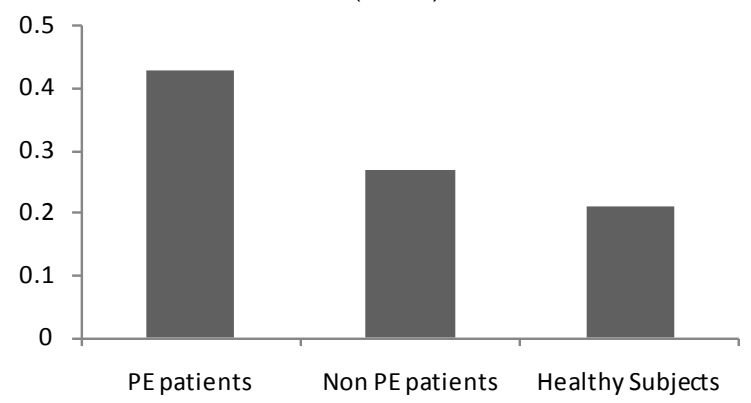

ABSU: absorbance units.

Figure 3. IMA mean levels among the subjects included in this study.

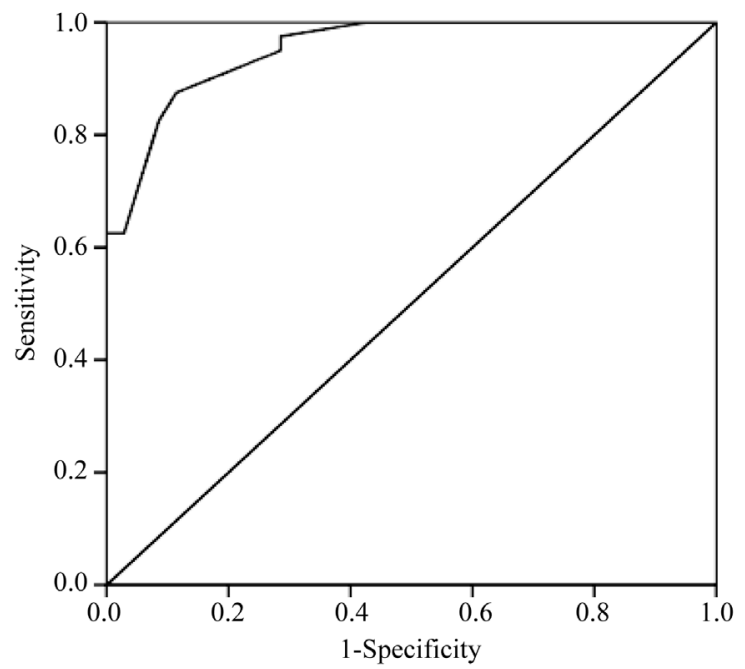

Figure 4. ROC curve for IMA in this study. The area under the curve was 0.952 considered to be excellent test.

\section{Discussion}

D-dimer testing was known for many years ago. It has a good role in the exclusion and the diagnosis of pulmonary embolism. In this study the mean values of D-dimer were statistically significantly higher among the PE patient group in comparison with non PE patient group and healthy control subjects. D-dimer was high at $78.66 \%$ of the patients and was normal at $21.34 \%$ of the patients. These results are in agreeing with that of many other investigators. King et al. [13] found that D-dimer results were positive in 171 patients (85\%). In a study carried out by Walter et al. [14] and included 65 patients with confirmed acute PE, hs-cTnI and D-dimer values were measured. D-dimer was high at $65 \%$ of the patients. Alnomasy et al. [15] found that $69.5 \%$ of patients had an elevated level of D-dimer and 30.5\% was normal D-dimer level. In a study by Turedi et al. [16] the mean D-dimer levels were $12.48 \pm 10.88 \mathrm{microg} / \mathrm{ml}$ for pulmonary embolism patients; $5.36 \pm 7.80 \mathrm{microg} / \mathrm{ml}$ for non pulmonary embolism patients and $0.36 \pm 0.16 \mathrm{mi}-$ 
$\mathrm{crog} / \mathrm{ml}$ for healthy control subjects. In spite of the role of D-dimer in the diagnosis of pulmonary embolism, its specificity is not high. D-dimer testing in our work had $97.50 \%$ sensitivity and $42.85 \%$ specificity. The positive predictive value of D-dimer in this study was $66.10 \%$ while the negative predictive value was $93.75 \%$. Other researchers found similar results. Waser et al. [17] detected D-dimer sensitivity 98\%, specificity 49\%, NPV 97\%, PPV 62\% and exclusion rate 28\%. King et al. [13] found that the NPV and sensitivity of D-dimer were $97 \%$ and 98\%, respectively while the specificity and PPV were $18 \%$ and $25 \%$, respectively.

IMA has been suggested as a promising marker for the identification of patients with hypoxemia and ischemia such as pulmonary embolism patients [3]. In our study the mean values of IMA were statistically significantly higher among the PE patient group in comparison with non PE patient group and healthy control subjects. At cut-off value of 0.305 ABSU, IMA had $97.5 \%$ sensitivity and $71.42 \%$ specificity. The area under the curve was 0.952 . The positive predictive value of this cut-off value was $79.59 \%$ while the negative predictive value was $96.15 \%$. In comparison with D-dimer, IMA has the same sensitivity but significantly better specificity (71.42\% for IMA vs $42.85 \%$ for D-dimer). Similar findings have been found by other investigators. A study by Turedi et al., [18] consisting of 30 patients with PE and 30 healthy individuals, demonstrated that serum IMA levels were significantly higher than those in healthy individuals in $97 \%$ of patients. In another study by Turedi et al. [16] consisted of 130 patients with suspected PE and 59 healthy controls. Mean IMA levels were $0.362 \pm 0.11$ ABSU for the PE group ( $n=75) ; 0.265 \pm 0.07$ ABSU for the non PE group $(n=55)$; and $0.175 \pm 0.05 \mathrm{ABSU}$ for the healthy control group. At a cut-off point of 0.25 ABSU, IMA was $93 \%$ sensitive and $75 \%$ specific in the diagnosis of PE. PPV was $79.4 \%$ and NPV was $78.6 \%$. Zheng et al. [19] found the levels of IMA (75.84 \pm 15.70 $\mathrm{U} / \mathrm{ml})$ and D-dimer $(5.41 \pm 5.29 \mathrm{mg} / \mathrm{l})$ in patients with acute pulmonary embolism (APE) were significantly higher than that in healthy controls. According to the ROC curve, the most appropriate IMA cut-off value in APE was $63.30 \mathrm{U} / \mathrm{ml}$ with sensitivity $87.2 \%$, specificity $80 \%$. The most appropriate D-dimer cut-off value in APE was $0.57 \mathrm{mg} / \mathrm{L}$ with sensitivity $94.9 \%$, specificity $66.7 \%$. The use of IMA in combination with D-dimer has a positive impact on the specificity value. The level of plasma IMA in high risk group of APE was higher significantly than that in medium or low risk groups. In a study carried out by Hogg et al. [20], 452 patients were investigated for DVT, and 354 patients were investigated for PE. 348 patients investigated for PE had IMA testing as did 195 of the first 199 DVT patients. VTE prevalence was 19.7\%. The IMA:albumin ratio performed better than
IMA alone. The area under the ROC curve (AUC) for IMA:albumin in all VTE was 0.60 (95\% CI 0.54 to 0.66), in DVT 0.56 (95\% CI 0.46 to 0.65$)$ and in PE 0.63 (95\% CI 0.56 to 0.71 ). In ED patients with symptoms of PE, the AUC for IMA:albumin was 0.69 (95\% CI 0.60 to 0.78).

Based on these results for IMA, we can suggest that it can be used as an alternative marker instead of D-dimer in the diagnosis and exclusion of pulmonary embolism. IMA has some advantages over D-dimer including: Better specificity, PPV and NPV, rapid technique, and lower cost.

\section{Conclusions}

IMA is a good alternative to D-dimer in the diagnosis and exclusion of PE. Larger studies are needed to augment our results.

Limitations: A small number of the subjects and selected patients criteria.

\section{REFERENCES}

[1] S. Ozsu, F. Oztuna, Y. Bulbul, et al. "The Role of Risk Factors in Delayed Diagnosis of Pulmonary Embolism," American Journal of Emergency Medicine, Vol. 29, No. 1, 2011, pp. 26-32. http://dx.doi.org/10.1016/j.ajem.2009.07.005

[2] V. F. Tapson, “Acute Pulmonary Embolism,” The New England Journal of Medicine, Vol. 358, No. 10, 2008, pp. 1037-1052. http://dx.doi.org/10.1056/NEJMra072753

[3] D. C. Gaze, "Ischemia Modified Albumin: A Novel Biomarker for the Detection of Cardiac Ischemia,” Drug Metabolism and Pharmacokinetics, Vol. 24, No. 4, 2009, pp. 333-341. http://dx.doi.org/10.2133/dmpk.24.333

[4] B. S. Hausen, C. Signor, H. Kober, E. Tatsch, R. S. Pereira, T. Duarte, C. L. Hermes, M. M. Duarte and R. N. Moresco, "Effect of Temperature on Albumin Cobalt Binding and Its Influence on Ischemia-Modified Albumin Levels in Patients with Suspected Acute Coronary Syndrome," Clinical Laboratory, Vol. 58, No. 1-2, 2012, pp. 169-172.

[5] K. Han, N. Jia, L. Yang and L. Q. Min, "Correlation between Ischemia-Modified Albumin and Lipid Levels in Patients with Acute Cerebrovascular Disease," Molecular Medicine Reports, Vol. 6, No. 3, 2012, pp. 621-624.

[6] M. Uygun, S. Yilmaz, M. Pekdemir, C. Duman and Y. S. Gürbüz, "The Diagnostic Value of Ischemia-Modified Albumin in a Rat Model of Acute Mesenteric Ischemia,” Academic Emergency Medicine, Vol. 18, No. 4, 2011, pp. 355-359.

http://dx.doi.org/10.1111/j.1553-2712.2011.01045.x

[7] K. Maneewong, T. Mekrungruang-wong, S. Luangaram, T. Thongsri and S. Kumphune, "Combinatorial Determination of Ischemia Modified Albumin and Protein Carbonyl in the Diagnosis of NonST-Elevation Myocardial Infarction,” Indian Journal of Clinical Biochemistry, Vol. 26, No. 4, 2011, pp. 389-395. 
http://dx.doi.org/10.1007/s12291-011-0118-2

[8] E. Kountana, K. Tziomalos, P. Semertzidis, F. Dogrammatzi, A. Slavakis, S. Douma, C. Zamboulis and P. Geleris, "Comparison of the Diagnostic Accuracy of Ischemia-Modified Albumin and Echocardiography in Patients with Acute Chest Pain,” Experimental \& Clinical Cardiology, Vol. 18, No. 2, 2013, pp. 98-100.

[9] A. Toker, A. Aribas, F. H. Yerlikaya, E. Tasyurek and K. Akbuğa, "Serum and Saliva Levels of Ischemia-Modified Albumin in Patients with Acute Myocardial Infarction," Journal of Clinical Laboratory Analysis, Vol. 27, No. 2, 2013, pp. 99-104. http://dx.doi.org/10.1002/jcla.21569

[10] W. Ghanima, M. Abdelnoor, M. C. Mowinckel and P. M. Sandset, "The Performance of STA-Liatest D-Dimer Assay in Out-Patients with Suspected Pulmonary Embolism," British Journal of Haematology, Vol. 132, No. 2, 2006, pp. 210-215. http://dx.doi.org/10.1111/j.1365-2141.2005.05859.x

[11] D. Bar-Or, E. Lau and J. Winker, "A Novel Assay for Cobalt-Albumin Binding and It's Potential as a Marker Formyocardial Ischemia-A Preliminary Report," The Journal of Emergency Medicine, Vol. 19, No. 4, 2000, pp. 311-315. http://dx.doi.org/10.1016/S0736-4679(00)00255-9

[12] A. van Belle, H. R. Buller, M. V. Huisman, P. M. Huisman, K. Kaasjager, P. W. Kamphuisen, M. H. Kramer, M. J. Kruip, J. M. Kwakkel-van Erp, F. W. Leebeek, M. Nijkeuter, M. H. Prins, M. Sohne and L. W. Tick, "Effectiveness of Managing Suspected Pulmonary Embolism Using an Algorithm Combining Clinical Probability, D-Dimer Testing, and Computed Tomography," JAMA, Vol. 295, No. 2, 2006, pp. 172-179. http://dx.doi.org/10.1001/jama.295.2.172

[13] V. King, A. A. Vaze, C. S. Moskowitz, L. J. Smith and M. S. Ginsberg, "D-Dimer Assay to Exclude Pulmonary Embolism in High Risk Oncologic Population: Correlation with CT Pulmonaryangiography in an Urgent Care Setting," Radiology, Vol. 247, No. 3, 2008, pp. 854-861. http://dx.doi.org/10.1148/radiol.2473070939
[14] T. Walter, P. Apfaltrer, F. Weilbacher, M. Meyer, S. O. Schoenberg, C. Fink and J. Gruettner, "Predictive Value of High-Sensitivity Troponin I and D-Dimer Assays for Adverse Outcome in Patients with Acute Pulmonary Embolism,” Experimental and Therapeutic Medicine, Vol. 5, No. 2, 2013, pp. 586-590.

[15] S. F. Alnomasy, S. Ansari, M. A. Izhari, S. Alam and S. Ali, "Evaluation of D-Dimer Level among Adult Patients in Al-Quwayyah Government Hospital, Saudi Arabia," Journal of Applied Pharmaceutical Science, Vol. 2, No. 12, 2012, pp. 82-84.

[16] S. Turedi, A. Gunduz, A. Mentese, M. Topbas, S. C. Karahan, S. Yeniocak, I. Turan, O. Eroglu, U. Ucar, Y. Karaca, S. Turkmen and R. M. Russell, "The value of Ischemia-Modified Albumin Compared with D-Dimer in the Diagnosis of Pulmonary Embolism,” Respiratory Research, Vol. 30, No. 9, 2008, p. 49. http://dx.doi.org/10.1186/1465-9921-9-49

[17] G. Waser, S. Kathriner and W. A. Wuillemin, "Performance of the Automated and Rapid STA Liatest D-Dimer on the STA-R Analyzer," Thrombosis Research, Vol. 116, No. 2, 2005, pp. 165-170. http://dx.doi.org/10.1016/j.thromres.2004.12.003

[18] S. Turedi, A. Gunduz, A. Mentese, S. C. Karahan, S. E. Yilmaz, O. Eroglu, I. Nuhoglu, I. Turan and M. Topbas, "The Value of Ischemia-Modified Albumin in the Diagnosis of Pulmonary Embolism," American Journal of Emergency Medicine, Vol. 25, No. 7, 2007, pp. 770-773. http://dx.doi.org/10.1016/j.ajem.2006.12.013

[19] L. B. Zheng, Z. Q. Wang, Z. Wang, M. Peng, J. T. Zhang, Z. He and L. G. Shi, "The Application of Ischemia Modified Albumin in the Diagnosis of Acute Pulmonary Embolism,” Journal of Clinical Cardiology, Vol. 9, No. 1, 2010, pp. 100-105.

[20] K. Hogg, E. Hinchliffe, S. Halsam, A. Valkov and F. Lecky, "Is Ischaemia-Modified Albumin a Test for Venous Thromboembolism?” Emergency Medicine Journal, Vol. 29, No. 6, 2012, pp. 455-459. 